phys. stat. sol. (b) 221, 77 (2000)

Subject classification: 71.36.+c; 78.47.+p; S7.12

\title{
Angular-Asymmetric Nonlinear Polariton Dynamics in Semiconductor Microcavities
}

\author{
P. G. SAvvidis (a), J. J. BAumberG ${ }^{1}$ ) (a), R. M. Stevenson (b), \\ M. S. Skolnick (b), J. S. Roberts (c), and D. M. WhitTAKeR (d)
}

(a) Department of Physics and Astronomy and Electronics and Computer Science, University of Southampton, Southampton SO17 1BJ, UK

(b) Department of Physics, University of Sheffield S3 7RH, UK

(c) Department of Electronic and Electrical Engineering, Sheffield S1 3JD, UK

(d) Toshiba Europe Research Ltd., Cambridge, CB4 4WE, UK

(Received April 10, 2000)

\begin{abstract}
Parametric interactions in semiconductor heterostructures in which two exciton quasiparticles mutually scatter are typically weak because they are restricted by energy-momentum conservation. Here, semiconductor microcavities in the strong coupling regime are optically pumped at specific resonant energy and angle of incidence to enormously enhance the parametric interactions. A femtosecond goniometer is devised to allow time-, wavelength- and polarisation-resolved pump-probe spectroscopy. Time-resolved images of the microcavity emission at different angles reveal a highly asymmetric luminescence distribution which switches on as the pump power is increased. Both signal and idler beams can be identified when a probe pulse initiates stimulated scattering of polaritons from a reservoir. The images show the rich variety of interactions produced by the coupling of light-matter modes, and the future promise of dispersion engineering.
\end{abstract}

Introduction With the advent of highly controlled semiconductor epitaxies, both electronic and photonic wavefunctions and their interactions can be specifically manipulated. This has been the basis of the immense success of vertical cavity surface-emitting semiconductor lasers (VCSELs), which couple quantum well emission with high efficiency into a single cavity photon mode. However, because of the large carrier density under operating conditions, the system shows weak coupling of light and matter and the dispersion relations of the electron-hole pairs and the cavity photons remain freeparticle like (quadratic). It is thus reasonable to treat carriers and photons as independent objects. The availability in recent years of heterostructures in which resonant photon and exciton modes can become strongly coupled changes this description profoundly, leading to the clear observation of mixed photon-exciton modes (polaritons). These exciton polaritons possess rather different emission and absorption properties to the bare quantum wells $[1,2]$, however, there is still strong discussion as to whether new physics operates in the low density linear regime since most experiments can be straightforwardly explained using a non-dispersive oscillator model [3 to 5]. At high occupation densities, it is possible to saturate the exciton oscillator strength, in which case the strong coupling regime is destroyed and the system can once again be simply

\footnotetext{
1) Author for correspondence: Tel.: +44(0) 238059 3911; Fax: +44(0) 238059 3910; e-mail: j.j.baumberg@soton.ac.uk
} 
described in terms of carriers and photons [6 to 8], or in the coherent regime by an $a c$ field dressed exciton transition $[9,10]$. In between these two limiting regimes, a number of groups have shown using $\mathrm{cw}$ excitation that the polariton emission can shift in emission wavelength and display superlinear increases in intensity when increasing the power of a non-resonant $\mathrm{cw}$ pump [11 to 14]. However, the mechanism for this anomolous behaviour has remained unproven. Recently using pulsed excitation we definatively showed that parametric scattering between exciton-polaritons can produce extremely large angle-dependent nonlinearities if the pump light is resonantly injected at a specific angle of incidence [15]. This leads us to believe that parametric scattering between polaritons is extremely important in semiconductor microcavies.

We demonstrate here that the new distorted dispersion relations of such quasiparticles can evoke entirely new scattering properties. The most significant difference to emission from quantum wells is the highly asymmetric angular distribution of photoluminescence from the sample, and the strong observed parametric scattering of polaritons. The simultaneous use of time-, angular- and energy-resolved non-degenerate pump-probe spectroscopy directly tracks the dynamics, and greatly helps identification of the interactions involved. Pump polaritons resonantly injected at a 'magic' angle of $16.5^{\circ}$, experience strongly enhanced parametric scattering due to the Coulomb interactions between carriers, giving rise to highly nonequilibrium emission. Such optical interactions are related to hyper-Raman scattering previously used to study polaritons in bulk materials [16], and here directly visible due to the microcavity geometry.

Experiment The sample studied here is a $3 \lambda / 2$ semiconductor microcavity grown by MOVPE and consists of two pairs of three $10 \mathrm{~nm} \mathrm{In}_{0.06} \mathrm{Ga}_{0.94} \mathrm{As}$ QWs in $10 \mathrm{~nm}$ GaAs barriers, surrounded by distributed Bragg reflectors (DBRs) [15]. The sample strip is oriented with the cavity thickness constant in the plane formed by the incident beams. The sample is held in a cold-finger, wide field-of-view cryostat at a temperature of $4 \mathrm{~K}$. Figure $1 \mathrm{~B}$ shows the dispersion relation of the two polariton modes with normal mode splitting of $\hbar \Omega \approx 7 \mathrm{meV}$ (at $\theta=0^{\circ}$ ). The strong coupling between the excitons and cavity mode leads to two new polariton branches with distorted non-quadratic dispersion relations. In particular the lower polariton branch possesses a strong energy dip near $\mathbf{k}=0$, which acts as a polariton trap, and is responsible for the interactions we observe.

Our experimental geometry concentrates on the resonant angular coupling between a pump pulse incident at $16.5^{\circ}$ and a normally-incident probe pulse (Fig. 1A). When the exciton $\left(\omega_{\mathrm{x}}\right)$ and photon $\left(\omega_{\text {cav }}\right)$ modes are degenerate at $\theta=0^{\circ}\left(\omega_{\mathrm{x}}=\omega_{\text {cav }}\right)$ this pump configuration generates stimulated scattering shown by arrows in Fig. 1B, in which pump polariton pairs at $\left(\omega_{\mathrm{p}}, \mathbf{k}_{\mathrm{p}}\right)$ end up at the 'signal' $\left(\omega_{\mathrm{LP}}, \mathbf{k}_{\mathrm{p}}=0\right)$ and 'idler' $\left(\omega_{\mathrm{x}}, 2 \mathbf{k}_{\mathrm{p}}\right)$ [15]. The stimulated behaviour of this process is seen from the enhancement in scattering rate as the final state is occupied. In order to inject and extract polaritons at specific $\left(\omega_{\mathrm{p}}, \mathbf{k}_{\mathrm{p}}\right)$, the optical pulses must be spectrally filtered and aligned along particular incident angles, without grossly modifying their time of arrival at the sample. To achieve this we constructed a femtosecond-stable goniometer which rotates under the center of the sample with arms for pump, probe and detection beams which each allow access to $\theta= \pm 80^{\circ}$. A $100 \mathrm{fs} \mathrm{Ti}: \mathrm{S}$ laser is used to generate the different $3 \mathrm{ps}$ pulses which are wavelength-shaped in the spectral domain using computer-controlled liquid crystal modulators. The pump powers are kept sufficiently low so that the exciton oscillator strength suffers no appreciable bleaching (as measured from time-resolved trans- 


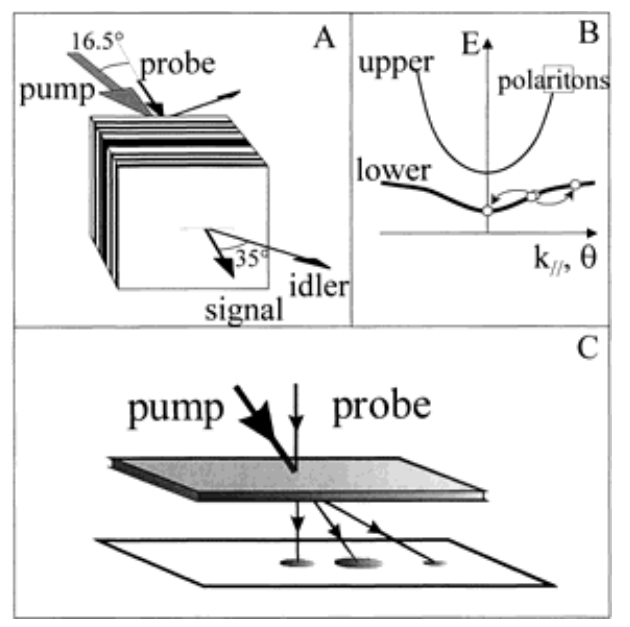

Fig. 1. A: Geometry of the microcavity with excitation pulse incident at $16.5^{\circ}$ and probe pulse at normal incidence producing signal gain from the probe and idler emission at $35^{\circ}$. B: Dispersion relation of upper and lower branch exciton-polaritons in the strong coupling regime. The horizontal axis is angle of incidence, which is roughly proportional to the in-plane polariton momentum. The arrows show the parametric scattering process between two pump polaritons described in the text. C: Geometry for imaging the emission on the screen

mission of the dips in reflectivity). Both pump and probe are co-circularly polarised as previously shown to give strongest interactions due to the exciton spin selection rules. The time-integrated emitted light from the sample can be resolved in angle, emission wavelength and polarisation. Here we image the emitted light from the sample on a screen to clearly show the asymmetry in the emission, using the transmission geometry shown in Fig. 1C.

Image Results The effect of the angular-dependent parametric gain can be clearly seen in this geometry. When only the pump pulse is incident, $80 \%$ of the incident light is reflected and the $12 \%$ transmitted is seen as the intense spot in the image in Fig. $2 \mathrm{a}$, where the horizontal axis corresponds to the angle of emission. At these powers, light also emerges at angles closer to the sample normal $\left(0^{\circ}\right.$ to $\left.20^{\circ}\right)$, however no light is seen at angles beyond $\theta<-2^{\circ}$. This confirms the non-thermalised nature of the emission under these resonant excitation conditions, since the potential minimum at $\mathbf{k}=0$ in the dispersion relation in Fig. $1 \mathrm{~b}$ is angular symmetric. Polaritons which scatter down from the injected pump energy, are immediately emitted before they have a chance to thermalise and lose the memory of the original direction of their in-plane momentum. To our knowledge, such photoluminescence asymmetries have not previously been identified. Figure $2 \mathrm{~b}$ shows the image when the probe pulse alone is normally incident, and this is dramatically modified when the pump pulse arrives at the same time (Fig. 2c). Strong amplification of the probe pulse (by a factor of twenty) is evident, while the emission from polaritons at angles between that of pump and probe is reduced. Definite confirmation of the parametric scattering is provided by the observation of an idler beam at the energy- and momentum-conserving angle of $35^{\circ}$, although this emission is weaker by a factor of ten [17] (the image in the region $\theta>25^{\circ}$ has been enhanced by a factor of two to show the idler more clearly). Previous nonlinear measurements in microcavities have found much weaker parametric scattering due to the nonresonant angular geometry employed [18, 19]. The spectral measurements also confirm the expected photoluminescence energy from the signal and idler polaritons. Idler polaritons are less likely to decay into photons because of their longer cavity lifetime compared to fast scattering with virtually degenerate high- $k$-excitons [17]. 

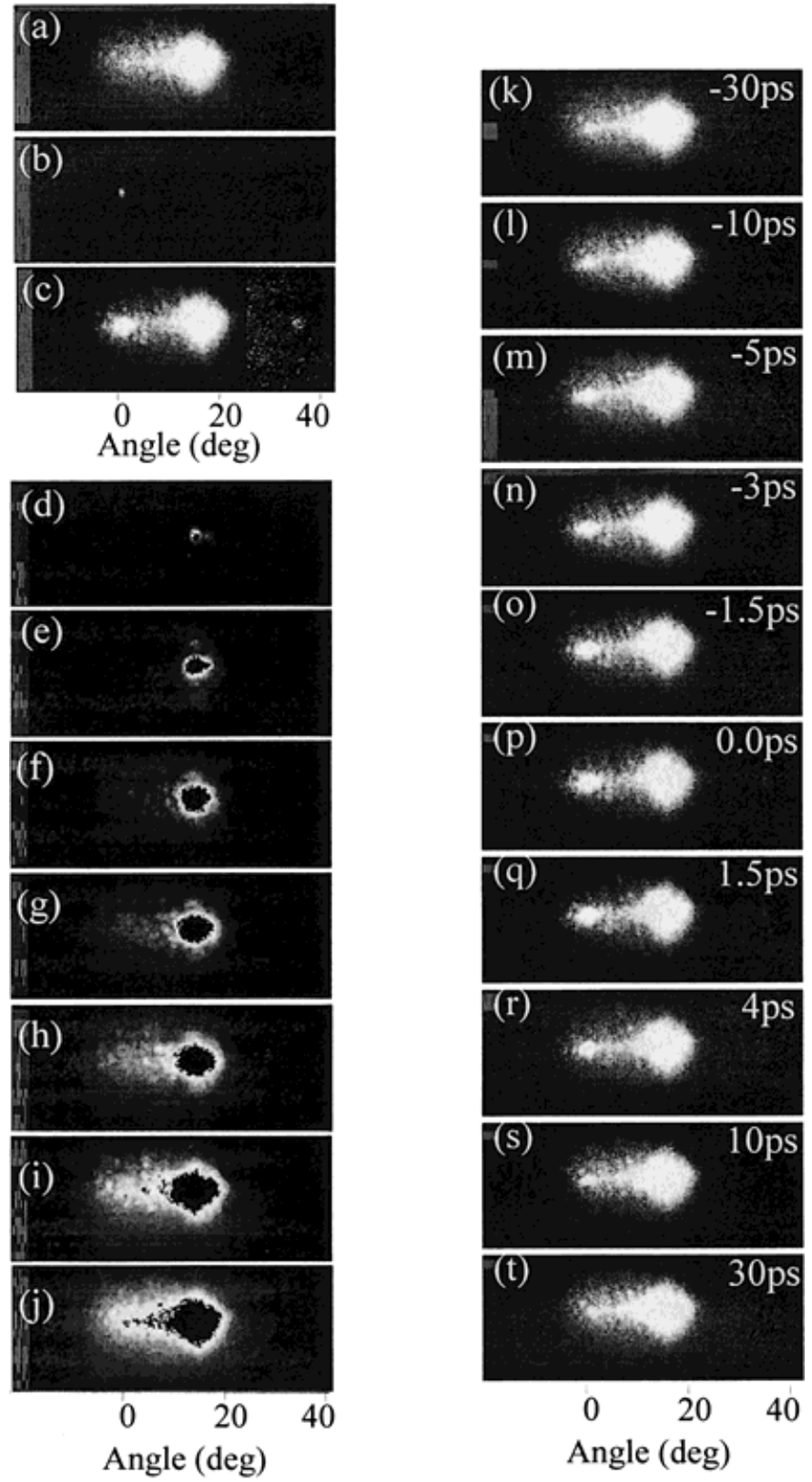

Fig. 2. Time-integrated images of the polariton emission at $T=4 \mathrm{~K}$, so that the horizontal axis corresponds to the emission angle. The intensities are on a linear gray scale, with the transmitted pump saturated in the image to bring out the PL. a) $20 \mathrm{~W} \mathrm{~cm}^{-2}$ pump pulse alone, incident at $16.5^{\circ}$, b) $100 \mathrm{~m} \mathrm{~W} \mathrm{~cm}{ }^{-2}$ probe pulse alone incident at $0^{\circ}$, c) pump and probe simultaneously arriving. Angles above $25^{\circ}$ have been enhanced by a factor of two to bring out the weak idler emission at $35^{\circ}$. d) to j) Power dependence of images for pump pulse alone at 2, 5, 8, 11, 14, 17, and $20 \mathrm{~W} \mathrm{~cm}^{-2}$ average pump powers. $\mathrm{k}$ ) to t) Emission images as a function of delay time between pump and probe pulses for conditions as in c) 
The strongly asymmetric emission is observed only when resonantly pumping polaritons near the turning point of the dispersion relation, $\theta \sim \sin ^{-1}\left\{\eta \sqrt{\frac{3 \Omega}{2 \omega_{\mathrm{x}}}}\right\}$, where the microcavity effective refractive index $\eta \approx 3.6$. At low pump powers, photoluminescence is extremely weak and most of the absorbed polaritons are reemitted back in the reflected and transmitted pump directions. As the pump power is increased (Figs. 2e to k), progressively more emission is seen towards (but not beyond) the sample normal. The nonlinear increase of this pulsed emission rules out dissapative scattering processes due to phonon emission, and is evidence for self-stimulated parametric scattering in which the first few polaritons which scatter into low energy states stimulate the scattering of further polaritons from the pump reservoir. We have recently confirmed this by analysing detailed spectra of the emission at different angles [17]. The lack of polariton luminescence at angles beyond the sample normal shows that there is no time for the polar-
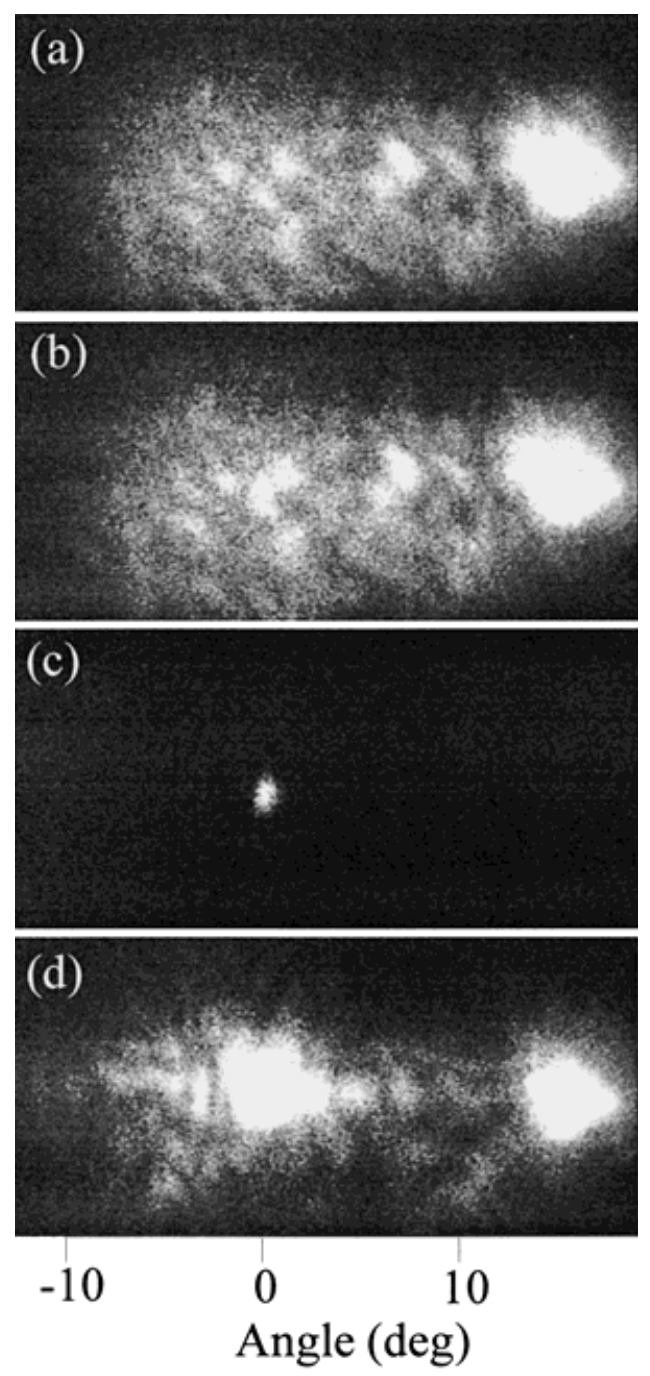

itons to thermalise before they escape from the cavity as photons.

The temporal dynamics of the parametric gain can be followed by changing the time of arrival of the probe pulse which stimulates the scattering from the reservoir of pump polaritons (Figs. $2 \mathrm{k}$ to t). Over the course of $5 \mathrm{ps}$, the gain rises smoothly, evolving from the self-stimulated emission pattern noted previously. Because the lifetime of polaritons at the pump energy is only a few ps, it is not yet possible to prove whether incoherent polaritons can provide the same parametric gain. However, it is well known [20] that this third-order interaction only constrains the sum of signal and idler phases to match twice the pump phase, $\phi_{\mathrm{s}}+\phi_{\mathrm{i}}=2 \phi_{\mathrm{p}}$. Hence for a specific probe phase to be amplified, any phase of pump polariton will suffice since the remaining

Fig. 3. Higher magnification time-integrated images of the polariton emission at an average pump power of $20 \mathrm{~W} \mathrm{~cm}^{-2}$. a), b) Pump pulse alone, taken at positions on the sample separated by $\approx 100 \mu \mathrm{m}$. c) Probe pulse alone of $100 \mathrm{~m} \mathrm{~W} \mathrm{~cm}^{-2}$. d) Pump and probe simultaneously arriving at the sample 
phase difference is given to the idler polariton. This is the basis for optical parametric oscillators pumped by multimode lasers. A further noteworthy feature is that although the probe pulse is an order of magnitude weaker than the luminescence at $0^{\circ}$, it is able to extract a much greater amount of energy from the pump polaritons than the selfstimulated emission. The origin of this effect must derive from the finite lifetime of the pump polaritons. By the time that a population is building up from spontaneous scattering into the $\mathbf{k}=0$ state, the pump polariton population is rapidly decreasing. On the other hand, the injected probe pulse is able to immediately initiate this stimulated scattering and thus exponentially enhances the final population in the $\mathbf{k}=0$ state.

The fine structure seen in all the images of Fig. 2 at angles smaller than the pump are reproducible, and highly dependent on the exact tuning conditions of the photon and exciton modes in the microcavity. In particular, bands of stronger emission can be tentatively identified at intervals of approximately $0.28 \mathrm{meV}$, suggesting the favouring of particular polariton decay channels with specific energy and momentum loss. These features are clearer in Fig. 3, which shows a magnified image of the spontaneous and stimulated scattering at a slightly different position on the sample. Further work is underway to identify this in detail and characterise their detuning dependence.

Summary In summary, we observe strong angular asymmetries in the emission from microcavities resonantly pumped at the turning point of the dispersion relation. Stimulated scattering traps significant signal populations of polaritons in the $\mathbf{k}=0$ state. In addition the parametric interactions generate idler polaritons at higher angles which can also be seen in the emission. Recently we have observed the cw parametric oscillation of these microcavities under $\mathrm{cw}$ excitation at the same angle [21]. The use of emission images helps identify the parametric regime, and clearly highlights the nonthermalised occupation of the lower polariton branch.

Acknowledgements We thank the support of the University of Southampton, Coherent UK Ltd., Hitachi Europe Ltd. and fruitful discussions with V. Savonna, B. Deveaud and C. Cuiti. This work was partly supported by EPSRC GR/M43890, GR/L32187, and HEFCE JR98SOBA.

\section{References}

[1] M. S. Skolnick, T. A. Fisher, and D. M. WhitTaker, Semicond. Sci. Technol. 13, 645 (1998).

[2] V. Savona, C. Piermarocchi, A. Quattropani, P. Schwendimann, and F. Tassone, in: New Aspects in Optical Properties of Nanostructures, Phase Transitions, Gordon \& Breach, New York/London 1998.

[3] C. Ell, J. Prineas, T. R. Nelson, JR., S. Park, H. M. Gibbs, G. Khitrova, S. W. Koch, and R. Houdré, Phys. Rev. Lett. 80, 4795 (1998).

[4] F. Quochi, C. Ciuti, G. Bongiovanni, A. Mura, M. Saba, U. Oesterle, M. A. Dupertuis, J. L. Staehli, and B. Deveaud, Phys. Rev. B 59, R15594 (1999).

[5] J. J. Baumberg, A. Armitage, M. S. Skolnick, and J. S. Roberts, Phys. Rev. Lett. 81, 661 (1998).

[6] A. Imamoglu, R. J. Ram, S. Pau, and Y. Yamamoto, Phys. Rev. A 53, 4250 (1996).

[7] J. K. RheE, D. S. Citrin, T. B. Norris, Y. Arakawa, and M. Nishioka, Solid State Commun. 97, 941 (1996).

[8] M. Kira, F. Jahnke, S. W. Koch, J. D. Berger, D. V. Wick, T. R. Nelson, Jr., G. Khitrova, and H. M. GibBs, Phys. Rev. Lett. 79, 5170 (1997).

X. Fan, H. Wang, H. Q. Hou, and B. E. Hammons, Phys. Rev. B 56, 15256 (1997). 
[9] F. Quochi, G. Bongiovanni, A. Mura, J. L. Staehli, B. Deveaud, R. P. Stanley, U. Oesterle, and R. Houdré, Phys. Rev. Lett. 80, 4733 (1998).

[10] F. Quochi, M. Saba, C. Ciuti, R. P. Stanley, R. Houdré, U. Oesterle, J. L. Staehli, and B. DeveAud, Phys. Rev. B 61, R5113 (2000).

[11] P. Snellart and J. Bloch, Phys. Rev. Lett. 82, 1233 (1999).

[12] Le si Dang, D. Heger, R. Andre, F. Boeuf, and R. Romestain, Phys. Rev. Lett. 81, 3920 (1998).

[13] S. Pau, H. Cao, J. Jacobson, G. Buörk, Y. Yamamoto, and A. Imamoglu, Phys. Rev. A 54, R1789 (1996).

[14] R. Huang, F. Tassone, and Y. Yamamoto, Phys. Rev. B 61, R7854 (2000).

[15] P. G. Savvidis, J. J. Baumberg, R. M. Stevenson, M. S. Skolnick, D. M. Whittaker, and J. S. RoBerts, Phys. Rev. Lett. 84, 1547 (2000).

[16] S. Savasta, R. Girlanda, and G. Martino, phys. stat. sol. (a) 164, 85 (1997), and references therein.

[17] P. G. Savvidis, J. J. Baumberg, R. M. Stevenson, M. S. Skolnick, D. M. Whittaker, and J. S. Roberts, submitted to Phys. Rev. B.

[18] M. Kuwata-Gonokami et al., Phys. Rev. Lett. 79, 1341 (1997). M. Shirane et al., Phys. Rev. B 58, 7978 (1998).

[19] F. Quochi, C. Ciuti, G. Bongiovanni, A. Mura, M. Saba, U. Oesterle, M. A. Dupertuis, J. L. Staehli, and B. Deveaud, Phys. Rev. B 59, R15594 (1999).

[20] Y. R. SHEN, The Principles of Nonlinear Optics, Wiley, New York 1984.

[21] J. J. Baumberg, P. G. Savvidis, R. M. Stevenson, M. S. Skolnick, D. M. Whittaker, A. I. TarTAKOVSKII, and J. S. RoBERTS, submitted to Nature. 
